Recepción: 20 / 04 / 2017

Aceptación: 20 / 05 / 2017

Publicación: 15 / 06 / 2017

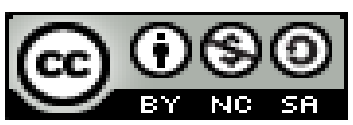

Ciencias Tecnológicas

Artículo de Investigación

\title{
Redes Sociales Informáticas como estrategias de aprendizaje en la escuela de Tecnología Médica de la Universidad de Guayaquil
}

\section{Informal Social Networks as learning strategies in the School of Medical Technology of the University of Guayaquil}

\section{Redes Sociais computador como aprender estratégias na Escola Superior de Tecnologia de Medicina da Universidade de Guayaquil}

\author{
Alfredo J. Veloz-Vélez ${ }^{\mathrm{I}}$ \\ alfredo.velozv@ug.edu.ec \\ Miguel A. Veloz-Montenegro II \\ miguel.velozm@ug.edu.ec \\ Raúl G. Castro-García III \\ raul.castrog@ug.edu.ec \\ Pedro A. Robles-Campos IV \\ pedro.roblesc@ug.edu.ec
}

Correspondencia: alfredo.velozv@ug.edu.ec

\footnotetext{
I. Ingeniero en Sistemas Administrativos Computarizados; Magister en Educación Informática; Docente Universidad de Guayaquil, Guayaquil, Ecuador.

II. Doctor en Medicina y Cirugía; Magister en Gerencia Clínica en Salud Sexual y Reproductiva; Docente Universidad de Guayaquil, Guayaquil, Ecuador.

III. Tecnólogo Medico en Terapia Respiratoria; Licenciado en Terapia Respiratoria; Magister en Diseño Curricular; Diploma Superior en Docencia Universitaria; Diploma Superior en Diseño Curricular por Competencias; Docente Universidad de Guayaquil, Guayaquil, Ecuador.

Iv. Licenciado en Imagenologia; Docente Universidad de Guayaquil, Guayaquil, Ecuador.
} 


\section{Resumen}

Durante los últimos años ha sido evidente la expansión de las redes sociales educativas, ha tenido una recogida muy grande y significativa en la educación de todos los niveles redes. A ganado un enorme espacio en tan poco tiempo, debido principalmente a la demanda de comunicación y colaboración entre los docentes y estudiantes, quienes forzaron por la rápida evolución de estas herramientas tecnológicas innovadora de la web y sus diferentes funciones, se vieron en la necesidad de cambiar sus modelos educativos, dejando de ser consumidor pasivo de información a ser activos productores y distribuidores de contenidos.

De ahí esto se orienta básicamente a la implementación de redes sociales al fortalecimiento en la formación académica de los terpistas, por lo cual el proceso investigativo se ha direccionado enfocado varios aspectos, con tener una visión muy clara de cómo estas constituyen un aporte al proceso de enseñanza aprendizaje y dentro de ello el papel de que debe desempeñar el docente de área humanística.

Desde luego que esta temática, que ha sido objeto de investigación surgió de la problemática de ¿Qué incidencia tiene la utilización las redes sociales para optimizar las estrategias y metodologías de aprendizaje en la carrera de Terapia Respiratoria?

Para solucionar y buscar alternativas al problema planteado, se estableció objetivos de manera clara y concisa en post de conducir la investigación mediante los procedimientos que permitan la obtención de los resultados esperados, demostrando que el proyecto es una herramienta que servirá de fuente para concienciar a docentes y discentes de las ventajas y desventajas de las Redes sociales educativas.

Palabras clave: Redes sociales educativas; Herramientas tecnológicas; modelos educativos; Fortalecimiento en la formación académica; proceso de enseñanza aprendizaje; ventajas y desventajas de las Redes sociales educativas. 


\section{Abstract}

During the last few years has been evident the expansion of social educational networks, has had a very large and significant collection in education at all levels networks. Having gained a huge space in such a short time, mainly due to the demand for communication and collaboration between teachers and students, who were forced by the rapid evolution of these innovative technological tools of the web and its different functions, were in need of To change their educational models, from being a passive consumer of information to being active producers and distributors of contents.

Hence this is basically oriented to the implementation of social networks to the strengthening in the academic training of the terpistas, reason why the investigative process has been focused focused several aspects, with a very clear vision of how they constitute a contribution to the process of Teaching learning and within it the role of the teacher of humanistic area.

Of course, this subject, which has been the subject of research, emerged from the problematic of What is the incidence of social networks to optimize strategies and learning methodologies in the career of Respiratory Therapy?

In order to solve and search for alternatives to the problem, it was established clear and concise objectives in post to conduct the research through the procedures that allow obtaining the expected results, demonstrating that the project is a tool that will serve as a source to raise awareness among teachers And learners of the advantages and disadvantages of educational social networks.

Key words: Educational social networks; Technological tools; Educational models; Strengthening of academic training; Teaching-learning process; Advantages and disadvantages of educational social networks 


\section{Resumo}

Nos últimos anos, a expansão da rede social educacional tem sido evidente, teve um muito grande e significativo na educação em todos os níveis de redes de coleta. A ganhou um enorme espaço em um tempo tão curto, eles principalmente devido à demanda por comunicação e colaboração entre professores e alunos, que foram forçados pelo rápido desenvolvimento dessas ferramentas tecnológicas inovadoras da web e suas diferentes funções, estavam na necessidade de alterar os seus modelos de ensino, deixando de ser consumidores passivos de informação a ser produtores activos e distribuidores de conteúdo.

Por isso, é basicamente visando a implementação de redes sociais para fortalecer a formação acadêmica de terpistas, de modo que o processo de investigação foi abordada focados vários aspectos, para ter uma visão muito clara de como estes constituem uma contribuição para o processo de aprender e ensinar dentro de si o papel a ser desempenhado pelo professor da área de humanista.

É claro que este assunto, que está sob investigação surgiu o problema da Qual o impacto que o uso de redes sociais para otimizar as estratégias de aprendizagem e metodologias carreira em terapia respiratória?

Para solucionar problemas e encontrar alternativas para o problema, os objetivos de forma clara e concisa em pós para conduzir pesquisas usando os procedimentos que irão produzir os resultados esperados foi estabelecida, demonstrando que o projeto é uma ferramenta que irá servir como uma fonte de sensibilizar os professores e estudantes das vantagens e desvantagens de redes sociais educacionais.

Palavras-chave: redes sociais educacionais; ferramentas tecnológicas; modelos educativos; O reforço da formação acadêmica; processo ensino-aprendizagem; vantagens e desvantagens de redes sociais educacionais. 


\section{Introducción.}

El uso de las herramientas informáticas (Castillo, 2008) especialmente las de comunicación se difunden cada vez más, el proceso de investigación con fines educativos se realiza atreves del internet, usando las Tics. Por ello se cree que es de fundamental el tener la claridad en los efectos de un proceso; este estudio permitirá tener argumentos para delimitar esta corriente de informatización que se da en el mundo y en el Ecuador

Otro punto importante para realizar este estudio es que normalmente el análisis que se hace de la incorporación de nuevas tecnologías (Cabero, 1994), ha sido realizado desde el punto de vista del proceso del aprendizaje, el proceso de gestión académica influye o no en la calidad educativa de terapistas respiratorios desde la percepción de sus actores y la valoración que tienen los médicos y terapistas de esa influencia.

En general para los estudios o análisis, se vincula tecnología de comunicación en lo educativo para el área de salud por ello se valoró su enfoque más allá del uso de sus herramientas informáticas desde un punto de vista de eficiencia educativa.

Puntualmente se ha iniciado con el estudio y aplicación de nuevas estrategias para mejorar la calidad de la institución y acortar las distancias entre el significado construido por las nuevas tecnologías y la realidad educativa (Díaz, 2008).

La importancia de este trabajo radica en el papel protagónico que tiene actualmente los médicos y terapista respiratorios por su capacidad de crear nuevas alternativas para el uso de la tecnología,

Visión General 
Una red social educativa puede definirse como un sitio web complejo que provee contenidos, servicios, soluciones técnicas y capacitación a docentes, estudiantes, representantes legales, funcionarios y demás miembros de la comunidad educativa con el fin de apoyar procesos de gestión educativa, de enseñanza y de aprendizaje.

Las redes sociales educativas nacionales de América Latina se destacan por origen, valores y función dentro del mundo de las tecnologías para la enseñanza. la inclusión digital es básica y consiste concretamente en fortalecer el trabajo con todos los médicos y terapistas respiratorios de un país trabajando por la equidad educativa y aportando a la calidad de los aprendizajes.

La Universidad es la fuente de los conocimientos científicos donde las alternativas de solución a los problemas de la comunidad, está obligado a revisar constantemente sus procesos de aprendizajes.

Las carreras profesionales deben responder a las necesidades sociales y económicas Actualmente las Universidades están empeñadas en proponer a los futuros profesionales carreras que les permitan desarrollar su creatividad y el aporte científico. La aceptación que tienen las carreras de la Escuela de Tecnología Médica de la Universidad de Guayaquil conlleva a que los docentes busquen nuevas metodologías de aprendizaje en los conocimientos científicos.

El docente debe ser el facilitador del aprendizaje, la tecnología ha permitido tener abundante información y es el docente quien debe incluir a seleccionar herramientas adecuadas para el aprendizaje activo. Ante este reto le corresponde a la Universidad presentar una propuesta de cambio que debe empezar por mejorar los procesos de aprendizaje que incluya al docente como referente para que se garanticen una educación universitaria de calidad y comunicación en múltiples problemas de la sociedad. 
En la Universidad de Guayaquil, se encuentra la Facultad de Ciencias Médicas y a su vez la Escuela de Tecnología Médica, que se encarga de promover la formación de Licenciados Médicos profesionales que en el futuro sean los responsables de la aplicación de la tecnología actualizada con una educación de calidad que conduzca al éxito de los futuros profesionales.

\section{Descripción del Proyecto}

Actualmente la comunicación en redes sociales se ha desarrollado muy ágilmente, pero en el área de salud no se ha caracterizado por generar habilidades de uso de herramientas informáticas de comunicación. Los profesionales en la salud se encuentran en una desventaja tecnológica dentro de la sociedad globalizada a nivel de comunicación.

La educación es una de las variables clave para explicar el proceso de creación y las perspectivas de desarrollo de los nuevos emprendimientos, los cuales tienen indudables efectos positivos sobre el desarrollo social en el que involucran procesos comunicacionales en los cuales la información, los mecanismos y los procesos para su transmisión se convierten en relaciones relevantes.

La comunicación humana se fundamenta básicamente en la percepción de nuestros sentidos, para que se lleve a cabo eficientemente la comunicación debe de ser presentada de una manera fácil y entendible como sonidos, gráficos de excelente resolución, videos, animaciones y comunicaciones a tiempo real, esto incrementa la retención de la información.

En los sistemas multimediales la información se dispone en nodos que forman redes, que permiten al usuario tener acceso a la información de una manera secuencial; en las redes sociales se propicia la aproximación lúdica hacia el conocimiento, llevando la característica desarrollo del 
entendimiento y la persuasión; en este sentido la posibilidad pedagógica ofrece al usuario posibilidades organizar y establecer las relaciones necesarias con la información, para aumentar o modificar conceptos previos.

Al docente le permite ocultar documentación electrónica o enlaces al instante, que se comunique fácilmente con los discentes y con uno en particular, estimula la participación, preguntas, interacción, así como facilita la ruptura del binomio docente-estudiante.

Roles más activos asumidos por discentes y docentes, se habla de roles activos ya que los discentes, principalmente, dejan de ser meros receptores del conocimiento para comenzar a ser constructores de nueva información. Ahora todos tienen las mismas oportunidades para ser totales protagonistas en la creación de nuevos conocimientos. el docente está más activo que nunca pues ya no solo analiza, procesa, selecciona la información; sino que también debe guiar, moderar, advertir, acompañar a los nuevos constructores del conocimiento.

\section{Descripción del problema del mundo real}

El siglo XXI caracterizado por la comunicación global, creación de nuevas tecnologías, el trabajo en equipo en búsqueda de la calidad, ha cambiado la visión que se tenía del mundo en los diferentes campos, especialmente en la educación, como un sitio web complejo que provee contenidos, servicios, soluciones técnicas y capacitación a docentes, estudiantes, representantes legales, con el fin de apoyar procesos de gestión educativa, de enseñanza y de aprendizaje.

Las redes sociales educativas nacionales de América Latina se destacan por origen, valores y función dentro del mundo de las tecnologías para la enseñanza. Nacieron y crecieron como iniciativas públicas, abiertas y gratuitas para promover la asistencia en el sistema educativo. 
Cada modelo educativo adoptado por la sociedad en sus diferentes épocas ha respondido a las necesidades de ese tiempo; sin embargo, en un momento determinado entran en crisis lo cual acelera los cambios y siendo la Universidad la fuente de conocimientos científicos donde las alternativas de solución a los problemas de la comunidad.

Las Universidades están empeñadas en proponer a los futuros profesionales carreras que les permitan desarrollar su creatividad y el aporte científico, presentando una propuesta de cambio que debe empezar por mejorar los procesos de aprendizaje que incluya al docente como referente para que se garanticen una educación universitaria de calidad.

\section{Materiales y métodos.}

Se ha planteado esta propuesta para ayudar a los docentes de la de la Facultad de Ciencias Médicas de la Universidad de Guayaquil a realizar técnicas que ayuden al inter- aprendizaje y al aprendizaje.

Las Redes sociales en la actualidad se han convertido en las mayores herramientas tecnológicas de comunicación, entretenimiento, investigación, el mismo que trata de abordar las redes en la educación, La realización de la guía académica, ha surgido de la necesidad de aprovechar esos conocimientos e insertarlos al proceso educativo, a través de la utilización de Redes Sociales, los docentes, quienes indudablemente son los llamados a promover el inter-aprendizaje, en donde por ningún motivo se debe desconocer las ventajas que hoy en día brinda la tecnología, mediante sus diferentes herramientas, dentro de las cuales constan las Redes Sociales Educativas.

Esta temática debe ser asumida con la integración de procedimientos claros y precisos, los mismos que estén debidamente relacionados con los propósitos planeados y con ello poder 
contribuir de una forma acertada al uso adecuado de las Redes anteriormente especificadas, fomentando un cambio positivo en la educación de los discentes de la Escuela de Tecnología Médica de la Universidad de Guayaquil.

Y aunque las aplicaciones son cada vez más sencillas de utilizar, y los estudiantes ya son nativos digitales, es necesario darles una formación en términos de alfabetización digital.

El presente trabajo de investigación se efectúa de manera acorde, ya que se cuenta con el apoyo de la comunidad educativa (autoridades, docentes y estudiantes), además porque la escuela cuenta con un laboratorio de cómputo, el mismo que posee el servicio de internet, estos medios ayudarán al cumplimiento de los objetivos planteados, que conlleven a desarrollar un adecuado proceso de enseñanza aprendizaje.

Con la aplicación de las Redes sociales en la educación, se promueve el uso de las Tics en el aula, desarrollando un aprendizaje dinámico y efectivo. Sin embargo, para que este aprendizaje se genere y sea exitoso, es fundamental saber enseñar a los estudiantes a trabajar en equipo, algo que además les será esencial en su futuro académico.

La guía académica expuesta es original, porque no existe otro tema similar al nuestro en la institución y específicamente a los años de estudio a la cual va dirigida. Por lo que se ha considerado elemental aplicar un proceso investigativo, con lineamientos eficaces que direccionen a dar solución al problema planteado y así alcanzar los objetivos esperados.

Las Tics se asocia a la dinámica de las redes sociales ya que permite el desarrollo de actividades de manera colaborativa y también promueve la integración de recursos en un entorno delimitado y personalizado. Es una aplicación que tiene la posibilidad de integrar en su interior otras 
aplicaciones y de publicar sus resultados en documentos públicos y blogs, por lo que tiene todas las características de la Web 3.0.

Se puede decir que, las herramientas tecnológicas también n se basan en el aprendizaje colaborativo y en las ideas de la Pedagogía constructivista que afirma que el conocimiento se construye en la mente del discente en lugar de ser transmitido a partir de libros o enseñanzas sin cambios.

La universalización de las redes sociales es un fenómeno consolidado. En Ecuador la utilización de las redes sociales no sólo para actividades lúdicas sino como herramientas de gestión del conocimiento en formación universitaria cada vez cobra más fuerza. Las redes sociales ofrecen un gran potencial para la puesta en marcha de proyectos de innovación docente que permitan la adquisición de competencias y destrezas transferibles en los estudiantes.

Las posibilidades educativas de las redes sociales son enormes, ya que se han convertido en un espacio idóneo para intercambiar información y conocimiento de una forma rápida, sencilla, pero es necesario despertar el interés y motivar al profesorado y al estudiantado.

Autores como de Haro (2009), atribuyen al uso de las redes sociales en el ámbito docente tres ventajas, independientemente de la asignatura de que se trate, del profesorado, o del grupo de discentes.

Minimizar la necesidad de formación porque todos usan el mismo recurso.

Favorecen la comunicación de manera bidireccional.

Su carácter generalista permite el uso universal de las mismas. 
En el ámbito universitario el uso de las redes sociales y herramientas como los blogs permiten ofrecer recursos didácticos que complementen los recursos tradicionales de los que disponen la educación universitaria. El profesorado debe potenciar el diseño y elaboración de entornos sociales virtuales interactivos, en los cuales el verdadero protagonista de los procesos formativos sea el estudiante.

\section{Método}

La metodología a utilizar es los criterios de utilización aplicación y experiencia de los docentes de la Escuela de Tecnología Médica en la aplicación de las redes sociales en las cátedras que imparten. Por esto se considera que el método es cualitativo.

Se utilizó las técnicas como entrevista y encuesta bajo un cuestionario de preguntas cerradas. Los instrumentos de la investigación se utilizaron los siguientes: Observación, que consiste en observar el hecho y registrar su análisis, está la encuesta que nos permite recolectar información clara y precisa, tenemos también la entrevista que es el acto comunicativo de que se establece con el agente que se dese tener información

Los temas a consultar son: conocimiento de redes sociales acceso a ellas, su aplicación a la catedra. Con este tipo de cuestionamientos se realiza su aplicación en estudios comparados cuando se procede e análisis de diferentes procesos o tendencias de la utilización de diversas tecnologías.

\section{Descripción de Población y Muestra}

La población de la investigación se encarga de todos los docentes de la Escuela de Tecnología Medicina de las cuatro carreras de habilitadas como Terapia Respiratoria, Terapia Ocupacional, Terapia de Lenguaje y Dietética y nutrición que es un equivalente a 62 docentes. 
Para la muestra se tomar la misma cantidad de docentes de la población para mayor eficiencia en los resultados estadísticos.

\begin{tabular}{|l|c|c|}
\hline \multicolumn{1}{|c|}{ Estratos } & Población & Muestra \\
\hline Docentes Contratados & 30 & 30 \\
\hline Docentes Nombramiento & 32 & 32 \\
\hline Total & 62 & 62 \\
\hline
\end{tabular}

Tabla 1. Proceso de Población y Muestra

\section{Resultados}

Nivel de conocimiento de los docentes sobre las Tecnología de Comunicación e información.

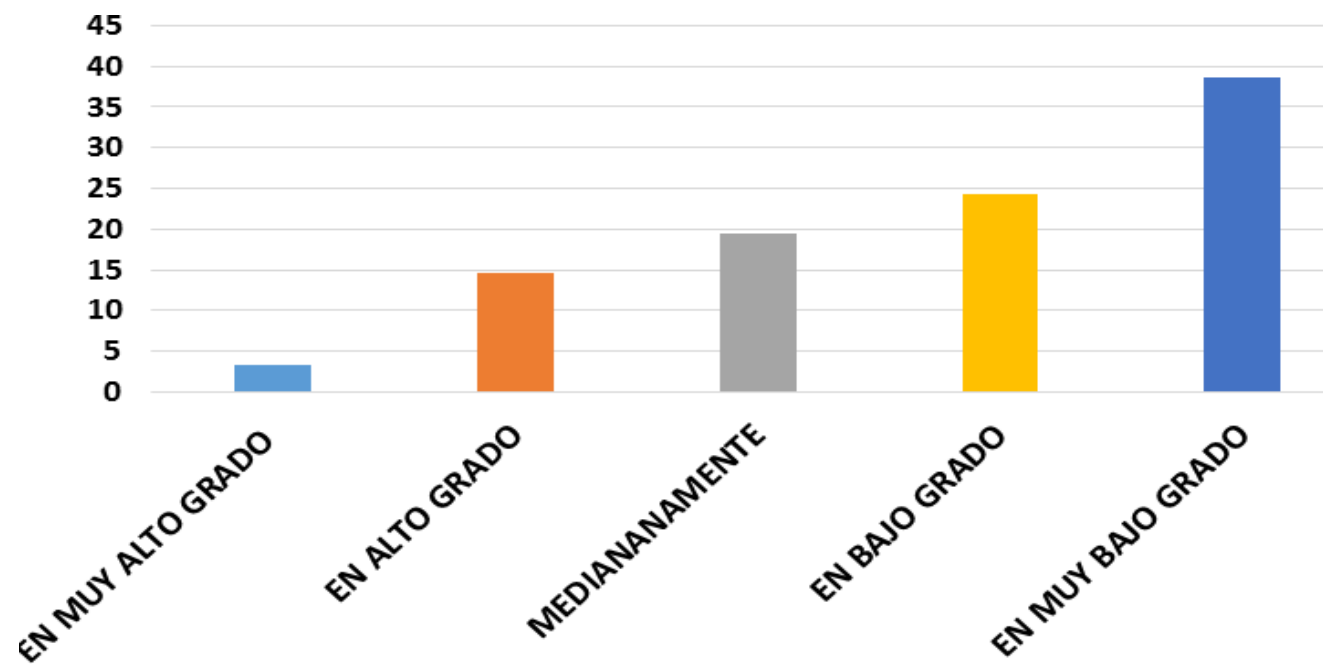

Grafico 1

Dificultad de los docentes para la aplicación de las Tics en el proceso de aprendizaje de su catedra. 


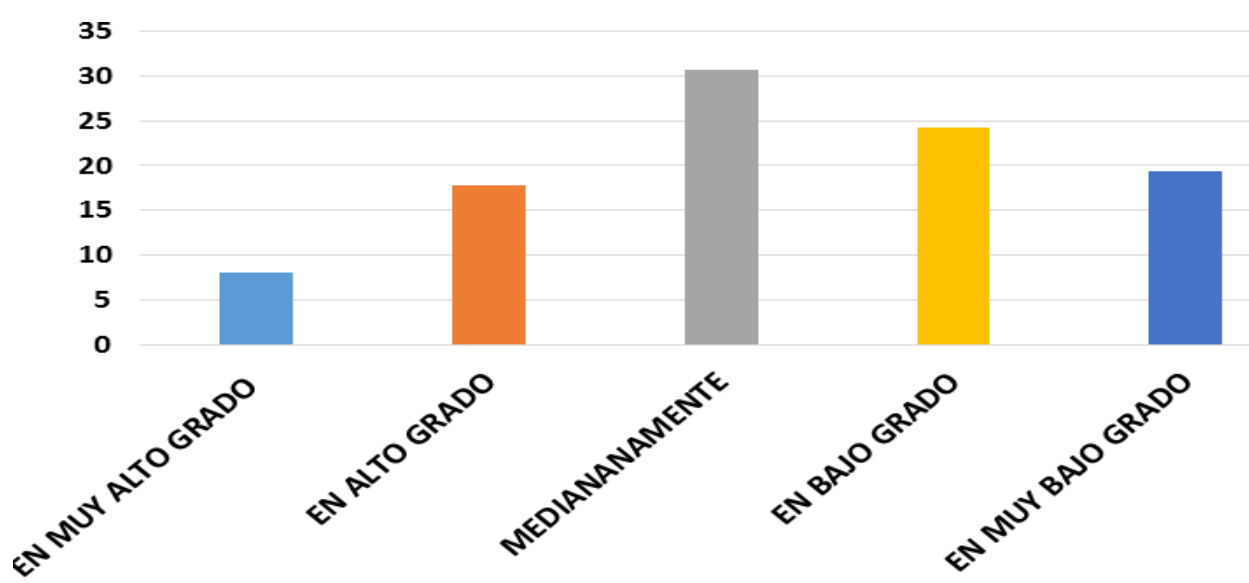

Grafico 2

Limitaciones de los docentes de la Escuela de Tecnología Médica para la aplicación de las Tics en su catedra.

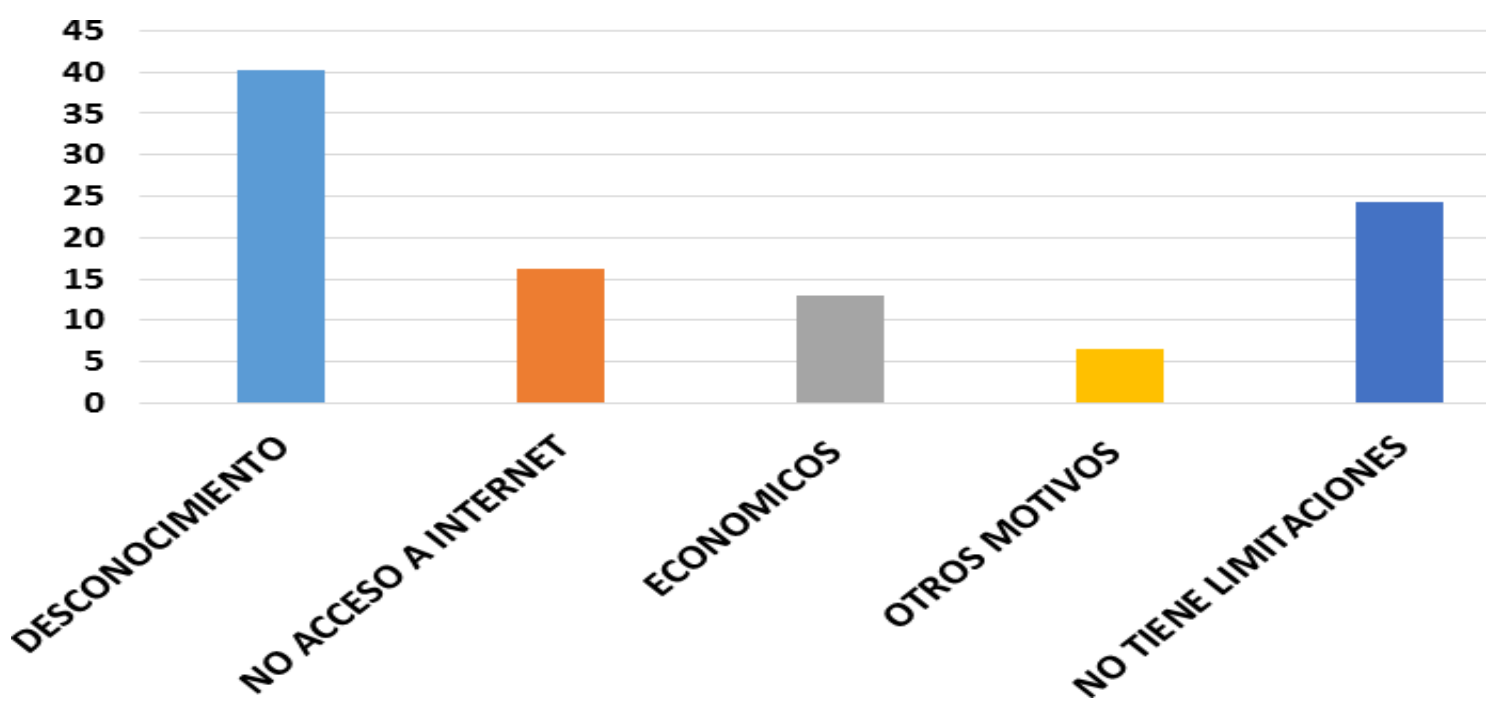

Grafico 3

Nivel de aceptación del docente de Tecnología Médica en utilizar una Guía Académica para la aplicación de las Tecnologías de Información y Comunicación como estrategia de aprendizaje. 
Redes Sociales Informáticas como estrategias de aprendizaje en la escuela de Tecnología Médica

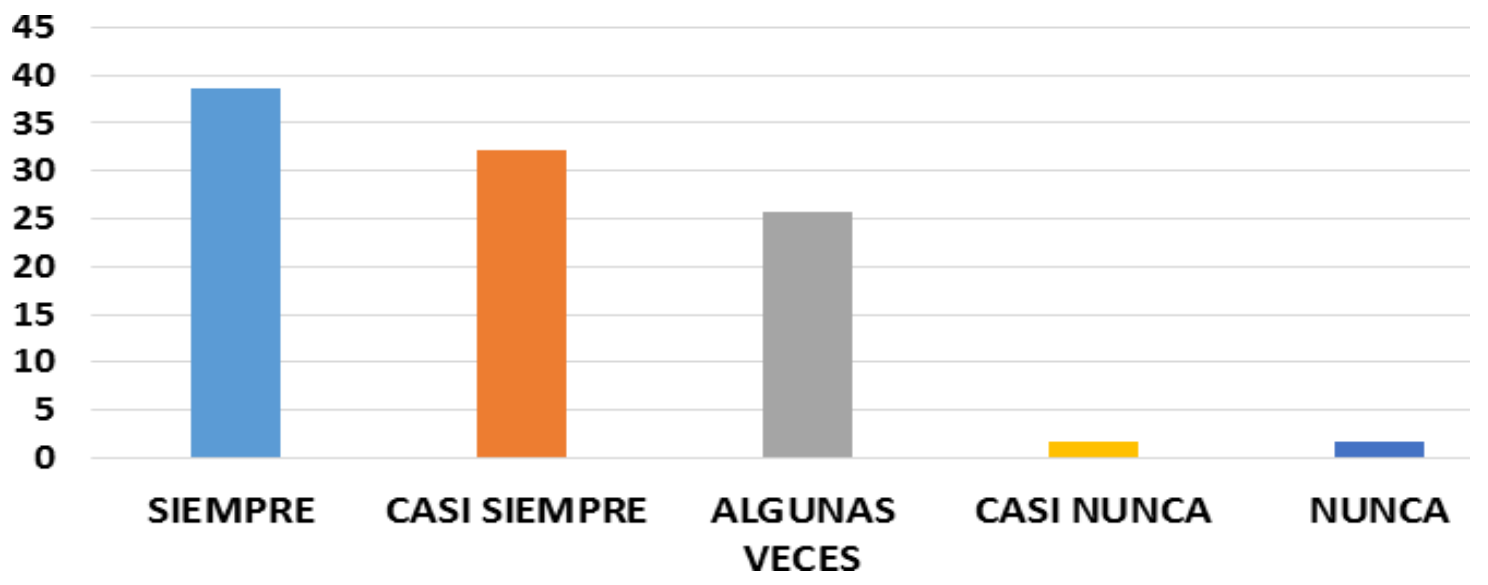

Grafico 4

\section{Conclusiones}

Se puede concluir que de acuerdo a la investigación realizada las redes sociales educativas se están convirtiendo en una herramienta que permite el desarrollo de las habilidades comunicativas y puede ser utilizada como estrategia de aprendizaje por parte de los docentes, pues la mayoría de ellos usan casi siempre, aunque consideran que lo hacen sin ningún beneficio educativo especifico, a pesar de que si se las incluye parcialmente como parte de sus actividades académicas.

Por otra parte, los estudiantes tienen la percepción de que los docentes no usan las redes sociales educativas, con el fin de ser utilizada en el proceso de enseñanza aprendizaje, pues solo las utilizan parcialmente para pronósticos de investigación de tipo general.

Se coincide con artero (2011) en que manejo de una red social en el aula puede suponer un acercamiento entre el docente y el discente. Las redes sociales se conciben desde y para la interacción y este nuevo espacio de dialogo puede ser un lugar de aprendizaje y enriquecimiento mutuo: por una parte, el docente interviene, modula y colabora en el aprendizaje con los discentes, 
incluso puede hacer uso de ellas para extraer la variedad de estilos de aprendizaje que conviven en su aula también la evaluación del comportamiento individual y colectivo de estudiantes.

Las redes sociales educativas pueden convertirse en una estrategia de aprendizaje, entendida como el conjunto de operaciones, pasos, rutinas que usan los discentes para facilitar la obtención almacenamiento, recuperación y uso de información al aprender.

De considerarse lo anterior, las redes sociales educativas contribuirla a una transformación educativa que permitirá un espacio de dialogo y propiciaría un aprendizaje y enriquecimiento mutuo entre los integrantes de la comunidad educativa; es decir, un sitio donde la interacción, enriquezca el aprendizaje colaborativo y fomente la meta cognición de los actores con actividades que ellos conlleven a la autoevaluación o reflexión sobre el trabajo realizado.

\section{Bibliografía}

Byrne, N., \& Rozental, M. (1994). Tendencias actuales de la educación médica y propuesta de orientación para la educación médica en América Latina. Educ Med Salud, 28(1), 53-93.

Cabero, J. (1994). Nuevas tecnologías, comunicación y educación. Redalyc.

Castillo, A. (2008). Herramientas informáticas para la aplicación de técnicas de desarrollo de pensamiento creativo. REDALYC.

Codina, L. (2009). Ciencia 2.0: Redes sociales y aplicaciones en línea para académicos. Hipertext. net, 7 , 295.

Huamaní, C., \& Mayta-Tristán, P. (2010). Producción científica peruana en medicina y redes de colaboración, análisis del Science Citation Index 2000-2009. Revista Peruana de Medicina Experimental y Salud Pública, 27(3), 315-325.

Torres, C. I., \& Alcántar, M. D. R. C. (2011). Uso de las redes sociales como estrategias de aprendizaje. \& Transformación educativa?. Apertura, 3(2), 6-15.

Moreno, M. P., Beltrán, C. A., Rodríguez, G. A., \& Estrada, P. R. (2006). Autoestima y redes sociales de apoyo como factores protectores de mobbing en docentes. Salus, 7(2).

Pérez, A. O. (2013). Postear retórica: análisis de caso de dos publicaciones en la comunidad virtual Taringa!. Revista Iberoamericana de Argumentación, (6). 
Redes Sociales Informáticas como estrategias de aprendizaje en la escuela de Tecnología Médica

Díaz, F. (2008). Educación y nuevas tecnologías de la información: ¿Hacia un paradigma educativo innovador? REDALYC. 